\title{
Selected abstracts in radio science
}

Radio Spectrum Utilization, A report published, for the Joint Technical Advisory Committee, by the Institute of Electrical and Electronics Engineers, Inc. [345 East 47th St., New York, 10017], 271 pages, 1964.

As indicated in the preface, "the book is intended primarily for the frequency administrator who is not necessarily a scientist." Nevertheless, sufficient background information is provided to give an appreciation of the technical factors involved.

The first chapter, which was prepared by the executive committee, presents the overall conclusions drawn from the detailed material in the rest of the book. In this chapter, the "technical ground rules" for spectrum allocations are introduced. Specific recommendations are then made for the various ranges of the frequency spectrum.

The second chapter is an historical survey, reviewing the development of the radio spectrum from the first regular use of wireless telegraphy to the present. It includes an interesting and informative discussion of the activities of various international conferences which deal with frequency allocations.

The third chapter, which is 170 pages in length, summarizes the present state of knowledge of radio wave propagation from the radio engineer's viewpoint. Some of the specific topics considered are: the propagation medium, antennas, radio noise, effects of nuclear explosions, system and propagation loss concepts, receiving systems, diversity effects, waveguide mode theory, ray treatment of propagation, groundwave propagation, general characteristics of skywave propagation, ionospheric soundings, tropospheric propagation, meteorological conditions, factors affecting earth-space communications, ionospheric scatter, meteor burst propagation, and sporadic $E$ propagation. It is interesting to note that extensive use is made of the concept of propagation loss which has the advantage of separating out antenna environmental factors from the effects of propagation.

It is unfortunate that some of the figures in chapter III have symbols which are not explained in the text. Furthermore, there appear to be a number of misprints, improper and incomplete reference citations, and some inconsistent notation between various portions of the chapter. Despite these minor shortcomings, the material taken as a whole is a useful and readable summary of a complex subject.

The fourth and final chapter presents an analysis of the present allocations of the radio spectrum. It includes recommendations for long-term changes in these allocations in order to achieve more effective use of the entire radio spectrum. Although there is no author index or subject index, there is fairly extensive bibliography at the end of the book.

(Review by J. R. Wait) 